\title{
NECESSARY AND SUFFICIENT CONDITION FOR OSCILLATIONS OF NEUTRAL DIFFERENTIAL EQUATIONS
}

\author{
M. R. S. KULENOVIĆ ${ }^{1,2}$, G. LADAS ${ }^{1}$ AND A. MEIMARIDOU ${ }^{1,3}$
}

(Received 13 December, 1985)

\begin{abstract}
Consider the neutral delay differential equation

$$
\frac{d}{d t}[y(t)+p y(t-\tau)]+\sum_{t=1}^{k} q_{t} y\left(t-\sigma_{t}\right)=0
$$

where $p \in \mathbf{R}, \tau \geqslant 0, q_{1}>0$, and $\sigma_{1} \geqslant 0$ for $i=1,2, \ldots, k$. We prove the following result.

THEOREM. A necessary and sufficient condition for the oscillation of all solutions of Eq. (1) is that the characteristic equation

$$
\lambda+p \lambda e^{-\lambda \tau}+\sum_{i=1}^{k} q_{i} e^{-\lambda \sigma} l=0
$$
\end{abstract}

has no real roots.

\section{Introduction}

Neutral delay differential equations (NDDE for short) are differential equations in which the highest order derivative of the unknown function is evaluated both at the present time $t$ and at some past time $t-\tau$.

\footnotetext{
${ }_{1}^{1}$ Department of Mathematics, University of Rhode Island, Kingston, R.I. 02881, USA.

${ }^{2}$ Department of Mathematics, University of Sarajevo,Sarajevo 71000, Yugoslavia.

${ }^{3}$ Department of Electrical Engineering, Democritus University of Thrace, Xanthi 67100, Greece.

(c) Copyright Australian Mathematical Society 1987, Serial-fee code 0334-2700/87
} 
In this paper we prove the following result:

\section{TheOREM. Consider the NDDE}

$$
\frac{d}{d t}[y(t)+p y(t-\tau)]+\sum_{i=1}^{k} q_{t} y\left(t-\sigma_{t}\right)=0,
$$

where $p \in \mathbf{R}, \tau \geqslant 0, q_{t}>0$, and $\sigma_{t} \geqslant 0$, for $i=1,2, \ldots, k$. Then a necessary and sufficient condition for the oscillation of all solutions of Eq. (1) is that the characteristic equation

$$
\lambda+p \lambda e^{-\lambda \tau}+\sum_{i=1}^{k} q_{i} e^{-\lambda \sigma_{l}}=0
$$

has no real roots.

The special case of this results when $k=1$ was proved by Sficas and Stavroulakis [7]. Also for $k=1$ and $\tau, \sigma, p$, and $q \in \mathbf{R}$ the above theorem was proved by Grove, Ladas, and Meimaridou [3]. When $p=0$ or $\tau=0$, the above result is due to Tramov [10]. For a simple proof see Ladas, Sficas and Stavroulakis [6].

There are drastic differences in the behavior of solutions of NDDEs compared to nonneutral equations. For example Snow [9] has shown, see also [8], that even though the characteristic roots of (2) may lie in the negative half-plane, it is still possible for (1) to have unbounded solutions. Our theorem establishes the fact that unlike stability, the oscillatory nature of the solutions of (1) is determined by the roots of the characteristic equation (2).

Several results concerning the oscillation and asymptotic behavior of the solutions of (1), when $k=1$, were recently obtained by Ladas and Sficas [5] and Grammatikopoulos, Grove and Ladas [2].

Neutral equations have applications in electric networks containing lossless transmission lines. Such networks arise in high speed computers where the lossless transmission lines are used to interconnect switching circuits. See [1] and [4].

Let $\rho=\max \left\{\tau, \sigma_{1}, \sigma_{2}, \ldots, \sigma_{k}\right\}$ and $t_{0} \in \mathbf{R}$. By a solution of (1), in the interval $\left[t_{0}, \infty\right)$, we mean a function $y \in C\left(\left[t_{0}-\rho, \infty\right)\right.$, R) such that $y(t)+p y(t-\tau)$ is continuously differentiable for $t \geqslant t_{0}$ and $y(t)$ satisfies (1) for all $t \geqslant t_{0}$. Using the method of steps, it follows that for every $\phi \in C\left(\left[t_{0}-\rho, t_{0}\right], \mathbf{R}\right)$ there exists a unique solution of (1) in the interval $\left[t_{0}, \infty\right)$ such that $y(t)=\phi(t)$ for $t_{0}-\tau \leqslant t$ $\leqslant t_{0}$. 
As usual, a solution of (1) is called oscillatory if it has arbitrarily large zeros and nonoscillatory if it is eventually positive or eventually negative.

In the sequel, for convenience, when we write a functional inequality, we shall mean that it holds for all sufficiently large values of the argument $t$.

\section{Proof of the theorem}

The proof of the necessity part of the theorem is quite simple. If it is false, the characteristic equation (2) would have a real root $\lambda_{0}$ and therefore (1) would have the nonoscillatory solution

$$
y(t)=e^{\lambda_{0} t} .
$$

But this contradicts the hypothesis that every solution of (1) oscillates.

On the other hand, the proof of the sufficiency part is quite involved and will be divided into several cases. Here the assumption is that (2) has no real roots. Set

$$
F(\lambda) \equiv \lambda+p \lambda e^{-\lambda \tau}+\sum_{i=1}^{k} q_{i} e^{-\lambda \sigma_{t}}
$$

Then $F(0)=\sum_{i=1}^{k} q_{t}>0$ and so $F(\lambda)>0$ for every $\lambda \in \mathbf{R}$. Without loss of generality we will assume that $0 \leqslant \sigma_{1}<\sigma_{2}<\cdots<\sigma_{k}$. Clearly,

$$
\text { if } p>0 \text { then } \tau<\sigma_{k} \text {. }
$$

Otherwise, $F(-\infty)=-\infty$ which is impossible. Also $F(+\infty)=F(-\infty)=+\infty$ and so there exists a positive constant $m$ such that for every $\lambda \in \mathbf{R}$,

$$
\lambda+p \lambda e^{-\lambda \tau}+\sum_{i=1}^{k} q_{i} e^{-\lambda \sigma_{\imath}} \geqslant m .
$$

Assume, for the sake of contradiction, that (1) has an eventually positive solution $y(t)$. Set

$$
z(t)=y(t)+p y(t-\tau) .
$$

Using arguments similar to those in [2] and [5] the following lemma is easily established.

LEMMA 1.

(a) Let $p \geqslant-1$. Then $z(t)$ decreases monotonically to zero and

$$
\lim _{t \rightarrow \infty} y(t)=0 \text {. }
$$

(b) Let $p<-1$. Then $z(t)$ decreases monotonically to $-\infty$ and

$$
\lim _{t \rightarrow \infty} y(t)=\infty
$$


(c) $z(t)$ is a differentiable solution of (1), that is,

$$
\dot{z}(t)+p \dot{z}(t-\tau)+\sum_{i=1}^{k} q_{i} z\left(t-\sigma_{1}\right)=0 .
$$

(d) Set

$$
w(t)=z(t)+p z(t-\tau) .
$$

Then $w(t)$ is a twice-differentiable solution of (5). Furthermore,

$$
w(t)>0 \text { and } \ddot{w}(t)>0 \text {. }
$$

In particular, it follows from Lemma $1(\mathrm{~d})$ that

$$
p \neq-1 \text {. }
$$

Otherwise,

$$
\dot{w}(t)-\dot{w}(t-\tau)+\sum_{i=1}^{k} q_{i} w\left(t-\sigma_{t}\right)=0,
$$

and so

$$
\dot{w}(t)-\dot{w}(t-\tau)<0
$$

which contradicts the fact that $\ddot{w}(t)>0$.

As we remarked in the introduction, when $p \tau=0$ the theorem reduces to a known result about nonneutral equations (see [6]). Thus we will assume that $p \tau \neq 0$.

In view of the above observations, in the sequel, we will assume that $\tau \neq 0$ and $p \in(-\infty,-1) \cup(-1,0) \cup(0, \infty)$.

Case 1. $p>0$.

Set

$$
v(t)=[y(t)+p y(t-\tau)]+\sum_{i=1}^{k-1} q_{i} \int_{t-\sigma_{k}}^{t-\sigma_{1}} y(s) d s,
$$

with the convention that for $k=1$ the sum in the above notation is zero. Then

$$
\dot{v}(t)=-\left(\sum_{i=1}^{k} q_{i}\right) y\left(t-\sigma_{k}\right)<0
$$

and so $v(t)$ is a positive and strictly decreasing function. Next, we claim that $v(t)$ is a differentiable solution of the equation

$$
\dot{v}(t)+p \dot{v}(t-\tau)+\sum_{i=1}^{k} q_{i} v\left(t-\sigma_{i}\right)=0 .
$$

The proof of this claim is a consequence of Lemma 1(c) and the following interesting result. 
Lemma 2. Let $y(t)$ be a solution of (1) for $t \geqslant t_{0}$ and let $\alpha$ and $\beta$ be any constants. Then

$$
x(t)=\int_{t-\alpha}^{t-\beta} y(s) d s
$$

is a solution of (1) for $t \geqslant t_{0}+\max \{\alpha, \beta\}$.

Proof. We have

$$
\begin{aligned}
\frac{d}{d t}[x(t)+ & p x(t-\tau)] \\
& =y(t-\beta)-y(t-\alpha)+p[y(t-\tau-\beta)-y(t-\tau-\alpha)] \\
& =[y(t-\beta)+p y(t-\beta-\tau)]-[y(t-\alpha)+p y(t-\alpha-\tau)] \\
& =\int_{t-\alpha}^{t-\beta} \frac{d}{d s}[y(s)+p y(s-\tau)] d s=\int_{t-\alpha}^{t-\beta}-\left[\sum_{t=1}^{k} q_{t} y\left(s-\sigma_{t}\right)\right] d s \\
& =-\sum_{t=1}^{k} q_{t} \int_{t-\sigma_{t}-\alpha}^{t-\sigma_{i}-\beta} y(s) d s=-\sum_{t=1}^{k} q_{t} x\left(t-\sigma_{t}\right)
\end{aligned}
$$

and the proof is complete.

Set

$$
v_{n}(t)=\left\{\begin{array}{l}
v(t), \quad n=0 \\
{\left[v_{n-1}(t)+p v_{n-1}(t-\tau)\right]+\sum_{i=1}^{k-1} q_{i} \int_{t-\sigma_{k}}^{t-\sigma_{i}} v_{n-1}(s) d s, \quad n=1,2, \ldots}
\end{array}\right.
$$

Then, for $n=1,2, \ldots$ we have

$$
\begin{gathered}
v_{n}(t)>0, \quad \dot{v}_{n}(t)<0, \quad \ddot{v}_{n}(t)>0, \\
\dot{v}_{n}(t)=-\left(\sum_{i=1}^{k} q_{1}\right) v_{n-1}\left(t-\sigma_{k}\right),
\end{gathered}
$$

and

$$
\dot{v}_{n}(t)+p \dot{v}_{n}(t-\tau)+\sum_{i=1}^{k} q_{t} v_{n}\left(t-\sigma_{t}\right)=0
$$

Set

$$
\Lambda_{n}=\left\{\lambda \geqslant 0: \dot{v}_{n}(t)+\lambda v_{n}(t) \leqslant 0\right\} .
$$

The proof will be accomplished by showing that $\Lambda_{n}$ has the following contradictory properties.

$\left(\mathrm{P}_{1}\right) \Lambda_{n}$ is a nonempty and bounded interval of nonnegative numbers. In particular, there exist numbers $\lambda_{1}$ and $\lambda_{2}$, independent of $n$, such that

$$
\lambda_{1} \in \Lambda_{n} \text { and } \lambda_{2} \notin \Lambda_{n} \text { for } n=1,2, \ldots
$$


$\left(\mathrm{P}_{2}\right)$ There is a positive number $\mu$, independent of $n$, such that

$$
\lambda \in \Lambda_{n} \text { with } \lambda \geqslant \lambda_{1} \Rightarrow(\lambda+\mu) \in \Lambda_{n+1} \quad \text { for } n=1,2, \ldots .
$$

We will need the following lemma from [6]

Lemma 3. Let $A$ and $\alpha$ be positive constants. Assume that $u(t)>0$ satisfies the delay inequality

$$
\dot{u}(t)+A u(t-\alpha) \leqslant 0 .
$$

Then

$$
u(t-\alpha)<B u(t)
$$

where $B=4 /(\alpha A)^{2}$.

From (7) and (9) we have,

$$
\dot{v}_{n}(t-\tau)+p \dot{v}_{n}(t-\tau)+q_{k} v_{n}\left(t-\sigma_{k}\right)<0
$$

or

$$
\dot{v}_{n}(t)+\frac{q_{k}}{1+p} v_{n}\left(t-\left(\sigma_{k}-\tau\right)\right)<0 .
$$

Thus,

$$
\dot{v}_{n}(t)+\frac{q_{k}}{1+p} v_{n}(t)<0
$$

which proves that

$$
\lambda_{1} \equiv \frac{q_{k}}{1+p} \in \Lambda_{n} .
$$

Applying Lemma 3 to (10) we find

$$
v_{n}(t-\alpha)<B v_{n}(t)
$$

where $\alpha=\sigma_{k}-\tau$ and $B=4(1+p)^{2} /\left(\alpha^{2} q_{k}^{2}\right)$. Integrating (8) from $t-\alpha$ to $t$ we get

$$
v_{n}(t)-v_{n}(t-\alpha)+\left(\sum_{t=1}^{k} q_{i}\right) \alpha v_{n-1}\left(t-\sigma_{k}\right)<0
$$

and so

$$
\left(\sum_{i=1}^{k} q_{i}\right) v_{n-1}\left(t-\sigma_{k}\right)<\frac{1}{\alpha} v_{n}(t-\alpha) .
$$

Hence, from (8), using (11) and (12), we obtain

$$
0=\dot{v}_{n}(t)+\left(\sum_{i=1}^{k} q_{i}\right) v_{n-1}\left(t-\sigma_{k}\right)<\dot{v}_{n}(t)+\frac{B}{\alpha} v_{n}(t),
$$


which proves that $\Lambda_{n}$ is bounded above by

$$
\lambda_{2} \equiv B / \alpha=4(1+p)^{2} /\left[\left(\sigma_{k}-\tau\right)^{3} q_{k}^{2}\right] .
$$

The proof of $\left(\mathrm{P}_{1}\right)$ is complete. We now turn to proving $\left(\mathrm{P}_{2}\right)$.

Let $\lambda \in \Lambda_{n}$ and set

$$
\phi_{n}(t)=e^{\lambda t} v_{n}(t)
$$

Then

$$
\dot{\phi}_{n}(t)=e^{\lambda t}\left[\dot{v}_{n}(t)+\lambda v_{n}(t)\right] \leqslant 0
$$

and so $\phi_{n}(t)$ is a decreasing function. Now for $\lambda \in \Lambda_{n}$ with $\lambda \geqslant \lambda_{1}$ and with

$$
\mu=m /\left[1+p e^{\lambda_{2} \tau}+\left(e^{\lambda_{2} \sigma_{k}} / \lambda_{1}\right) \sum_{l=1}^{k-1} q_{i}\right]
$$

we have, using (3) and (4),

$$
\begin{aligned}
& \dot{v}_{n+1}(t)+(\lambda+\mu) v_{n+1}(t) \\
& =-\left(\sum_{i=1}^{k} q_{i}\right) v_{n}\left(t-\sigma_{k}\right)+(\lambda+\mu) v_{n}(t)+(\lambda+\mu) p v_{n}(t-\tau) \\
& +(\lambda+\mu) \sum_{i=1}^{k-1} q_{i} \int_{t-\sigma_{k}}^{t-\sigma_{i}} v_{n}(s) d s \\
& \leqslant e^{-\lambda t}\left[-\left(\sum_{i=1}^{k} q_{i}\right) e^{\lambda \sigma_{k}} \phi_{n}\left(t-\sigma_{k}\right)+(\lambda+\mu) \phi_{n}(t)\right. \\
& \left.+(\lambda+\mu) p e^{\lambda \tau} \phi_{n}(t-\tau)+(\lambda+\mu) \sum_{i=1}^{k-1} q_{t} e^{\lambda t} \int_{t-\sigma_{k}}^{t-\sigma_{i}} \phi_{n}(s) e^{-\lambda s} d s\right] \\
& \leqslant e^{-\lambda t} \phi_{n}\left(t-\sigma_{k}\right)\left[-\left(\sum_{i=1}^{k} q_{i}\right) e^{\lambda \sigma_{k}}+(\lambda+\mu)\right. \\
& \left.+(\lambda+\mu) p e^{\lambda \tau}+(\lambda+\mu) \sum_{i=1}^{k-1} q_{i}\left(e^{\lambda \sigma_{k}}-e^{\lambda \sigma_{i}}\right) / \lambda\right] \\
& =e^{-\lambda{ }^{\prime}} \phi_{n}\left(t-\sigma_{k}\right)\left[-\sum_{i=1}^{k} q_{i} e^{\lambda \sigma_{i}}+\lambda+\lambda p e^{\lambda \tau}\right. \\
& \left.+\mu\left(1+p e^{\lambda \tau}+\frac{1}{\lambda} e^{\lambda \sigma_{k}} \sum_{i=1}^{k-1} q_{i}\right)-\frac{\mu}{\lambda} \sum_{i=1}^{k-1} q_{i} e^{\lambda \sigma_{1}}\right] \\
& \leqslant e^{-\lambda t^{\prime}} \phi_{n}\left(t-\sigma_{k}\right)\left[-m+\mu\left(1+p e^{\lambda_{2} \tau}+\frac{1}{\lambda_{1}} e^{\lambda_{2} \sigma_{k}} \sum_{i=1}^{k-1} q_{t}\right)\right]=0
\end{aligned}
$$

which proves $\left(\mathrm{P}_{2}\right)$. The proof of the theorem in Case 1 is complete. 
Case 2. $-1<p<0$.

Set

$$
z_{n}(t)= \begin{cases}z(t)=y(t)+p y(t-\tau) & \text { if } n=0, \\ z_{n-1}(t)+p z_{n-1}(t-\tau) & \text { if } n=1,2, \ldots\end{cases}
$$

Then, in view of Lemma 1(a), (c), and (d), for each $n=1,2, \ldots$ we have

$$
\begin{gathered}
z_{n}(t)>0, \quad \dot{z}_{n}(t)<0, \quad \ddot{z}_{n}(t)>0, \\
\dot{z}_{n}(t)=-\sum_{i=1}^{k} q_{\imath} z_{n-1}\left(t-\sigma_{\imath}\right),
\end{gathered}
$$

and

$$
\dot{z}_{n}(t)+p \dot{z}_{n}(t-\tau)+\sum_{i=1}^{k} q_{i} z_{n}\left(t-\sigma_{i}\right)=0
$$

Set

$$
\Lambda_{n}=\left\{\lambda \geqslant 0: \dot{z}_{n}(t)+\lambda z_{n}(t) \leqslant 0\right\} .
$$

As in Case 1, it suffices to establish that $\Lambda_{n}$ has the contradictory properties $\left(\mathrm{P}_{1}\right)$ and $\left(\mathrm{P}_{2}\right)$.

Clearly, $\lambda_{1} \equiv 0 \in \Lambda_{n}$ for $n=1,2, \ldots$ Next, we will prove that $\Lambda_{n}$ is bounded above. From (13) and (15) we find,

$$
\dot{z}_{n}(t)+q_{k} z_{n}\left(t-\sigma_{k}\right)<0
$$

which, in view of Lemma 2, implies that

$$
z_{n}\left(t-\sigma_{k}\right)<\tilde{B} z_{n}(t),
$$

where

$$
\tilde{B}=4 /\left(\sigma_{k} q_{k}\right)^{2} \text {. }
$$

Note that if $\sigma_{k}=0$ then Eq. (1) has $k=1$ and this case has been covered in [3]. So, without loss of generality, we will assume that $\sigma_{k}>0$. Now, integrating (14) from $t-\alpha$ to $t, \alpha>0$ we get

$$
z_{n}(t)-z_{n}(t-\alpha)+\sum_{i=1}^{k} q_{i} \int_{t-\alpha}^{t} z_{n-1}\left(s-\sigma_{i}\right) d s=0,
$$

or

$$
\sum_{i=1}^{k} q_{i} z_{n-1}\left(t-\sigma_{i}\right)<\frac{1}{\alpha} z_{n}(t-\alpha) .
$$

Hence, from (14), using (16), (17) and $\alpha=\sigma_{k}$ we obtain

$$
0=\dot{z}_{n}(t)+\sum_{i=1}^{k} q_{t} z_{n-1}\left(t-\sigma_{\imath}\right)<\dot{z}_{n}(t)+\frac{\tilde{B}}{\sigma_{k}} z_{n}(t),
$$


which proves that

$$
\lambda_{2} \equiv \tilde{B} / \sigma_{k}=4 /\left(\sigma_{k}^{3} q_{k}^{2}\right)
$$

is an upper bound of $\Lambda_{n}$. The proof of $\left(P_{1}\right)$ is complete. Next, we will prove $\left(\mathrm{P}_{2}\right)$ with $\mu=m$. Let $\lambda \in \Lambda_{n}$ and set

$$
\psi_{n}(t)=e^{\lambda t} z_{n}(t)
$$

Then

$$
\dot{\psi}_{n}(t)=e^{\lambda t}\left[\dot{z}_{n}(t)+\lambda z_{n}(t)\right] \leqslant 0
$$

and so $\psi_{n}(t)$ is a decreasing function. Finally, for $\lambda \in \Lambda_{n}$ and using (4), we find

$$
\begin{aligned}
& \dot{z}_{n+1}(t)+(\lambda+m) z_{n+1}(t) \\
& =-\sum_{i=1}^{k} q_{i} z_{n}\left(t-\sigma_{l}\right)+(\lambda+m) z_{n}(t)+p(\lambda+m) z_{n}(t-\tau) \\
& =e^{-\lambda t}\left[-\sum_{i=1}^{k} q_{i} e^{\lambda \sigma_{i}} \psi_{n}\left(t-\sigma_{i}\right)+(\lambda+m) \psi_{n}(t)+p(\lambda+m) e^{\lambda \tau} \psi_{n}(t-\tau)\right] \\
& \leqslant e^{-\lambda \tau}\left[-\sum_{i=1}^{k} q_{i} e^{\lambda \sigma_{i}}+\lambda+p \lambda e^{\lambda \tau}+m\right] \psi_{n}(t) \leqslant e^{\lambda t}(-m+m) \psi_{n}(t) \\
& =0,
\end{aligned}
$$

which proves $\left(\mathrm{P}_{2}\right)$ and completes the proof of the theorem in Case 2.

Case 3. $p<-1$.

This case will be divided into the following two subcases:

Subcase 3(i). $\sigma_{k} \leqslant \tau$. Set

$$
u_{n}(t)= \begin{cases}-z(t)=-\{y(t)+p y(t-\tau)] & \text { if } n=0 \\ -\left[u_{n-1}(t)+p u_{n-1}(t-\tau)\right] & \text { if } n=1,2, \ldots\end{cases}
$$

Then, in view of Lemma 1(b), (c), and (d), $u_{n}$ satisfies the following properties for each $n=1,2, \ldots$ :

$$
\begin{gathered}
u_{n}(t)>0, \quad \dot{u}_{n}(t)>0, \quad \ddot{u}_{n}(t)>0, \\
\dot{u}_{n}(t)=\sum_{i=1}^{k} q_{i} u_{n-1}\left(t-\sigma_{i}\right),
\end{gathered}
$$


and

$$
\dot{u}_{n}(t)+p \dot{u}_{n}(t-\tau)+\sum_{i=1}^{k} q_{i} u_{n}\left(t-\sigma_{i}\right)=0
$$

Set

$$
\Lambda_{n}=\left\{\lambda \geqslant 0:-\dot{u}_{n}(t)+\lambda u_{n}(t) \leqslant 0\right\} .
$$

As in Case 1, it suffices to establish that $\Lambda_{n}$ has the properties $\left(\mathrm{P}_{1}\right)$ and $\left(\mathrm{P}_{2}\right)$. Clearly $\lambda_{1} \equiv 0 \in \Lambda_{n}$ for $n=1,2, \ldots$. We have,

$$
u_{n-1}(t-\tau)=\frac{u_{n}(t)+u_{n-1}(t)}{-p}
$$

and so (19) yields

$$
\begin{aligned}
\dot{u}_{n}(t) & =\frac{1}{-p} \sum_{i=1}^{k} q_{i}\left[u_{n}\left(t+\tau-\sigma_{i}\right)+u_{n-1}\left(t+\tau-\sigma_{i}\right)\right] \\
& >\frac{1}{-p} \sum_{i=1}^{k} q_{i} u_{n-1}\left(t+\tau-\sigma_{i}\right) .
\end{aligned}
$$

Integrating from $t-\tau$ to $t$ we obtain

$$
u_{n}(t)-u_{n}(t-\tau)>\frac{1}{-p} \sum_{t=1}^{k} q_{i} \int_{t-\tau}^{t} u_{n-1}\left(s+\tau-\sigma_{t}\right) d s
$$

and so

$$
\sum_{i=1}^{k} q_{i} u_{n-1}\left(t-\sigma_{i}\right)<\frac{-p}{\tau} u_{n}(t)
$$

From (21) and (19) we find

$$
-\dot{u}_{n}(t)+\left(\frac{-p}{\tau}\right) u_{n}(t)>0
$$

which proves that $\Lambda_{n}$ is bounded above by $\lambda_{2} \equiv-p / \tau$.

Let $\lambda \in \Lambda_{n}$ and set $\phi_{n}(t)=e^{-\lambda t} u_{n}(t)$. Then

$$
\dot{\phi}_{n}(t)=e^{-\lambda t}\left[\dot{u}_{n}(t)-\lambda u_{n}(t)\right]=-e^{-\lambda t}\left[-\dot{u}_{n}(t)+\lambda u_{n}(t)\right] \geqslant 0
$$

and so $\phi_{n}(t)$ is an increasing function. 
For $\lambda \in \Lambda_{n}$ and $\mu=m /(-p)$ we have

$$
\begin{aligned}
& -\dot{u}_{n+1}(t)+(\lambda+\mu) u_{n+1}(t) \\
& =-\sum_{i=1}^{k} q_{i} u_{n}\left(t-\sigma_{i}\right)-(\lambda+\mu) u_{n}(t)-p(\lambda+\mu) u_{n}(t-\tau) \\
& =e^{\lambda t}\left[-\sum_{i=1}^{k} q_{i} e^{-\lambda \sigma_{t}} \phi_{n}\left(t-\sigma_{i}\right)-(\lambda+\mu) \phi_{n}(t)-p(\lambda+\mu) e^{-\lambda \tau} \phi_{n}(t-\tau)\right] \\
& \leqslant e^{\lambda t} \phi_{n}\left(t-\sigma_{k}\right)\left[-\sum_{i=1}^{k} q_{i} e^{-\lambda \sigma_{t}}-\lambda-\mu-p \lambda e^{-\lambda \tau}-p \mu e^{-\lambda \tau}\right] \\
& \leqslant e^{\lambda t} \phi_{n}\left(t-\sigma_{k}\right)\left(-m+m e^{-\lambda \tau}\right) \leqslant 0,
\end{aligned}
$$

which completes the proof of the theorem in this subcase.

Subcase 3(ii). $\tau<\sigma_{k}$. Let $j=\min \left\{i: \tau<\sigma_{1}\right\}$. That is, $j=1$ if $\tau<\sigma_{1}$ and $j=\nu$ if $\sigma_{\nu-1} \leqslant \tau<\sigma_{\nu}$ for some $\nu=2,3, \ldots, k$. Set

$$
w_{n}(t)=\left\{\begin{array}{l}
-[y(t)+p y(t-\tau)]+\sum_{i=j}^{k} q_{i} \int_{t-\sigma_{t}}^{t-\tau} y(s) d s \quad \text { if } n=0, \\
-\left[w_{n-1}(t)+p w_{n-1}(t-\tau)\right]+\sum_{i=j}^{k} q_{i} \int_{t-\sigma_{t}}^{t-\tau} w_{n-1}(s) d s \quad \text { if } n=1,2, \ldots
\end{array}\right.
$$

Then, one can prove that for each $n=1,2, \ldots$ the following are true:

$$
\begin{gathered}
w_{n}(t)>0, \quad \dot{w}_{n}(t)>0, \quad \ddot{w}_{n}(t)>0, \\
\dot{w}_{n}(t)=\sum_{i=1}^{j-1} q_{i} w_{n-1}\left(t-\sigma_{i}\right)+\left(\sum_{i=j}^{k} q_{i}\right) w_{n-1}(t-\tau),
\end{gathered}
$$

and

$$
\dot{w}_{n}(t)+p \dot{w}_{n}(t-\tau)+\sum_{i=1}^{k} q_{i} w_{n}\left(t-\sigma_{i}\right)=0,
$$

with the convention that when $j=1$ the first sum in (23) is zero.

Set

$$
\Lambda_{n}=\left\{\lambda \geqslant 0:-\dot{w}_{n}(t)+\lambda w_{n}(t) \leqslant 0\right\} .
$$

As in Case 1, we will prove that $\Lambda_{n}$ has the contradictory properties $\left(\mathrm{P}_{1}\right)$ and $\left(P_{2}\right)$. We have,

$$
w_{n}(t)<-p w_{n-1}(t-\tau)+\sum_{i=j}^{k} q_{i}\left(\sigma_{i}-\tau\right) w_{n-1}(t-\tau)
$$


or

$$
-w_{n}(t) /\left[p+\sum_{i=j}^{k} q_{i}\left(\tau-\sigma_{i}\right)\right]<w_{n-1}(t-\tau) .
$$

Hence, from (23)

$$
\begin{aligned}
& 0 \geqslant-\dot{w}_{n}(t)+\left(\sum_{i=j}^{k} q_{i}\right) w_{n-1}(t-\tau) \\
&>-\dot{w}_{n}(t)+\left[-\sum_{i=j}^{k} q_{i}\right] w_{n}(t) /\left[p+\sum_{i=j}^{k} q_{i}\left(\tau-\sigma_{i}\right)\right]
\end{aligned}
$$

which proves that

$$
\lambda_{1} \equiv-\sum_{i=j}^{k} q_{i} /\left[p+\sum_{i=j}^{k} q_{i}\left(\tau-\sigma_{i}\right)\right] \in \Lambda_{n} \quad \text { for } n=1,2, \ldots
$$

Next we will prove that $\Lambda_{n}$ is bounded above. From (24) we find

$$
\dot{w}_{n}(t)+p \dot{w}_{n}(t-\tau)<0
$$

and using (23) we obtain

$$
\sum_{i=1}^{j-1} q_{i} w_{n-1}\left(t-\sigma_{i}\right)+\left(\sum_{i=j}^{k} q_{i}\right) w_{n-1}(t-\tau)+p \dot{w}_{n}(t-\tau)<0
$$

or

$$
w_{n}(t)+\frac{1}{p} \sum_{i=1}^{j-1} q_{i} w_{n-1}\left(t+\tau-\sigma_{i}\right)+\frac{1}{p}\left(\sum_{i=j}^{k} q_{i}\right) w_{n-1}(t)>0 .
$$

Integrating from $t-\tau$ to $t$ we get

$$
w_{n}(t)-w_{n}(t-\tau)+\frac{1}{p} \sum_{i=1}^{j-1} q_{i} \tau w_{n-1}\left(t-\sigma_{i}\right)+\frac{1}{p}\left(\sum_{i=j}^{k} q_{i}\right) \tau w_{n-1}(t-\tau)>0
$$

and so

$$
\sum_{i=1}^{j-1} q_{i} w_{n-1}\left(t-\sigma_{i}\right)+\left(\sum_{i=j}^{k} q_{i}\right) w_{n-1}(t-\tau)<\frac{-p}{\tau} w_{n}(t) .
$$

Therefore (23) yields

$$
-\dot{w}_{n}(t)+\frac{-p}{\tau} w_{n}(t)>0
$$

which proves that $\lambda_{2} \equiv-p / \tau$ is an upper bound of $\Lambda_{n}$.

Let $\lambda \in \Lambda_{n}$ and set $\phi_{n}(t)=e^{-\lambda t} w_{n}(t)$. Then

$$
\dot{\phi}_{n}(t)=-e^{-\lambda t}\left[-\dot{w}_{n}(t)+\lambda w_{n}(t)\right] \geqslant 0
$$

and so $\phi_{n}(t)$ is an increasing function. 
Now for $\lambda \in \Lambda_{n}$ with $\lambda \geqslant \lambda_{1}$ and with $\mu=1 /\left(-p+\frac{1}{\lambda_{1}} \sum_{i=1}^{k} q_{\imath}\right)$ we have, $-\dot{w}_{n+1}(t)+(\lambda+\mu) w_{n+1}(t)$

$$
\begin{aligned}
=- & \sum_{i=1}^{j-1} q_{i} w_{n}\left(t-\sigma_{i}\right)-\left(\sum_{i=j}^{k} q_{i}\right) w_{n}(t-\tau)-(\lambda+\mu) w_{n}(t) \\
& -(\lambda+\mu) p w_{n}(t-\tau)+(\lambda+\mu) \sum_{t=j}^{k} q_{i} \int_{t-\sigma_{i}}^{t-\tau} w_{n}(s) d s \\
= & e^{\lambda_{t}}\left[-\sum_{i=1}^{j-1} q_{i} e^{-\lambda \sigma_{i}} \phi_{n}\left(t-\sigma_{i}\right)-\left(\sum_{i=j}^{k} q_{i}\right) e^{-\lambda \tau} \phi_{n}(t-\tau)-(\lambda+\mu) \phi_{n}(t)\right.
\end{aligned}
$$$$
\left.-(\lambda+\mu) p e^{-\lambda \tau} \phi_{n}(t-\tau)+(\lambda+\mu) \sum_{i=j}^{k} q_{i} e^{-\lambda t} \int_{t-\sigma_{i}}^{t-\tau} e^{\lambda s} \phi_{n}(s) d s\right]
$$$$
\leqslant e^{\lambda t} \phi_{n}(t-\tau)\left[-\sum_{i=1}^{j-1} q_{i} e^{-\lambda \sigma_{l}}-\left(\sum_{i=J}^{k} q_{l}\right) e^{-\lambda \tau}-(\lambda+\mu)\right.
$$$$
\left.-(\lambda+\mu) p e^{-\lambda \tau}+(\lambda+\mu) \sum_{i=j}^{k} q_{l}\left(e^{-\lambda \tau}-e^{-\lambda \sigma_{i}}\right) / \lambda\right]
$$$$
=e^{\lambda t} \phi_{n}(t-\tau)\left[-\sum_{i=1}^{k} q_{i} e^{-\lambda \sigma_{s}}-\lambda-\lambda p e^{-\lambda \tau}-\mu-\mu p e^{-\lambda \tau}\right.
$$

$$
\begin{aligned}
&\left.+\frac{\mu}{\lambda}\left(\sum_{i=j}^{k} q_{i}\right) e^{-\lambda \tau}-\frac{\mu}{\lambda} \sum_{i=1}^{k} q_{i} e^{-\lambda \sigma_{i}}\right] \\
& \leqslant e^{\lambda t} \phi_{n}(t-\tau)\left[-m+\mu\left(-p+\frac{1}{\lambda_{1}} \sum_{i=1}^{k} q_{t}\right)\right] \\
&=e^{\lambda t} \phi_{n}(t-\tau)(-m+m)=0
\end{aligned}
$$

which completes the proof of the theorem in this subcase. The proof of the theorem is complete.

\section{References}

[1] R. K. Brayton and R. A. Willoughby, "On the numerical integration of a symmetric system of difference-differential equations of neutral type," J. Math. Anal. Appl. 18 (1967), 182-189.

[2] M. K. Grammatikopoulos, E. A. Grove and G. Ladas, "Oscillation of first order neutral delay differential equations", J. Math. Anal. Appl. (to appear).

[3] E. A. Grove, G. Ladas and A. Meimaridou, "A necessary and sufficient condition for the oscillation of neutral equations", J. Math. Anal. Appl. (to appear). 
[4] J. Hale, Theory of functional differential equations, (Springer-Verlag, New York, 1977).

[5] G. Ladas and Y. G. Sficas, "Oscillations of neutral delay differential equations," Canad. Math. Bull. (to appear).

[6] G. Ladas, Y. G. Sficas and I. P. Stavroulakis, "Necessary and sufficient conditions for oscillations," Amer. Math. Monthly 90 (1983), 637-640.

[7] Y. G. Sficas and I. P. Stavroulakis, "Necessary and sufficient conditions for oscillations of neutral differential equations", Proc. Int. Conf. on Theory and Applic. of Differential Equations, Pan American University, Edinburg, Texas 78539, USA, May 20-23, 1985.

[8] M. Slemrod and E. F. Infante, "Asymptotic stability criteria for linear systems of differential equations of neutral type and their discrete analogues", J. Math. Anal. Appl. 38 (1972), 399-415.

[9] W. Snow, "Existence, uniqueness, and stability for nonlinear differential-difference equations in the neutral case," N.Y.U. Courant Inst. Math. Sci. Rep. IMM-NYU 328, (February 1965).

[10] M. I. Tramov, "Conditions for oscillatory solutions of first order differential equations with a delayed argument," Izv. Vyssh. Uchebn. Zaved. Mat. 19 (1975), 92-96. 